OPEN ACCESS

Edited by:

Noha M. Mesbah,

Suez Canal University, Egypt

Reviewed by:

Robert Kourist,

Ruhr-University Bochum, Germany

Skander Elleuche,

Hamburg University of Technology,

Germany

*Correspondence:

Jennifer A. Littlechild

j.a.littlechild@exeter.ac.uk

Specialty section:

This article was submitted to Process and Industrial Biotechnology, a

section of the journal Frontiers in Bioengineering and Biotechnology

Received: 18 August 2015 Accepted: 28 September 2015

Published: 13 October 2015

Citation:

Littlechild JA (2015) Enzymes from extreme environments and their industrial applications.

Front. Bioeng. Biotechnol. 3:161. doi: 10.3389/fbioe.2015.00161

\section{Enzymes from extreme environments and their industrial applications}

\author{
Jennifer A. Littlechild* \\ Exeter Biocatalysis Centre, Biosciences, College of Life and Environmental Sciences, University of Exeter, Exeter, UK
}

This article will discuss the importance of specific extremophilic enzymes for applications in industrial biotechnology. It will specifically address those enzymes that have applications in the area of biocatalysis. Such enzymes now play an important role in catalyzing a variety of chemical conversions that were previously carried out by traditional chemistry. The biocatalytic process is carried out under mild conditions and with greater specificity. The enzyme process does not result in the toxic waste that is usually produced in a chemical process that would require careful disposal. In this sense, the biocatalytic process is referred to as carrying out "green chemistry" which is considered to be environmentally friendly. Some of the extremophilic enzymes to be discussed have already been developed for industrial processes such as an L-aminoacylase and a $\gamma$-lactamase. The industrial applications of other extremophilic enzymes, including transaminases, carbonic anhydrases, dehalogenases, specific esterases, and epoxide hydrolases, are currently being assessed. Specific examples of these industrially important enzymes that have been studied in the authors group will be presented in this review.

Keywords: $\gamma$-lactamase, L-aminoacylase, transaminase, carbonic anhydrase, epoxide hydrolase, esterase, dehalogenase

\section{INTRODUCTION}

A problem with using enzymes for industrial biotransformation reactions is often their inherent stability to the conditions employed. During industrial processes, the enzymes are often exposed to a different environment to their natural conditions within the cell such as non-natural substrates, high substrate concentrations, non-aqueous conditions, and extremes of $\mathrm{pH}$. When using non-natural substrates, enzymes from different classes can be found to accept the same substrate such as the case with racemic $\gamma$-lactam described below. Enzymes can also carry out "side reactions" when used at a different $\mathrm{pH}$ than that found inside the cell.

The availability of new genome sequences makes the search for new industrial enzymes a relatively easy process. Also the isolation of metagenomes from extremophilic sources provides DNA from potentially uncultivatable organisms. However, the identification of specific enzymes from this resource is only as good as the current bioinformatic analyses and many novel or unknown activities can be missed. It is therefore important to also screen genomic libraries against substrates of commercial interest for specific biocatalytic activities especially if turnover of a non-natural substrate is required.

The diversity which "nature" provides for extremophilic environments of temperature, $\mathrm{pH}$, acidity, alkalinity, pressure, and salinity can be exploited to discover new novel and potentially robust enzymes that are better suited for use in industrial applications. The increased number 
of extremophilic genomes and metagenomes that can now be sequenced by next-generation sequencing technologies provides an ever expanding resource for identification of new enzymes.

The mechanism of protein stabilization under extreme conditions varies depending on the microbial species and level of adaption required for survival in the host organism. For the acidophiles and alkalophiles, it is only the proteins exported from the cell that have to be stable under the extreme $\mathrm{pHs}$ of the growth environment, since the proteins inside the cell do not have to withstand these extreme conditions as the intracellular $\mathrm{pH}$ is maintained around $\mathrm{pH}$ 5.0-6.0. Some general features of enzyme stability have been observed from the analysis of the three-dimensional structures of enzymes isolated from extreme environments of high temperatures have been reviewed by Littlechild et al. (2013).

Many archaeal and bacterial enzymes isolated from extremophiles have general applications in molecular biology such as the hyperthermophilic Pyrococcus polymerase enzyme which has improved fidelity in PCR reactions when compared to thermophilic bacterial polymerase enzymes. Other thermophilic enzymes are of great importance to the breakdown of biomass and other materials such as waste plastics in order to contribute to a circular economy where nothing is wasted.

This review will concentrate on several specific examples of interest to the author where extremophilic enzymes are currently playing an important role as biocatalysts for the pharmaceutical and fine chemical industries.

\section{SPECIFIC EXAMPLES}

\section{The Sulfolobus solfataricus $\gamma$-Lactamase Enzyme}

An enzyme from the thermophilic archaeon Sulfolobus solfataricus MT4 can use the bicyclic synthon (rac)- $\gamma$-lactam (2-azabicyclo[2.2.1] hept-5-en-3-one) as a substrate to obtain a single enantiomer of the $\gamma$-bicyclic lactam product which is an important building block for the anti-HIV compound, Abacavir (Taylor et al., 1993). This (+)- $\boldsymbol{\gamma}$-lactamase was identified in the Sulfolobus strain by screening colonies from an expression library for their ability to produce the amino acid product when supplied with the racemic $\gamma$-lactam. Screening was carried out using genomic libraries using a filter paper overlay. The colonies on the plate that were active showed a brown coloration of the filter paper when the amino acid was produced which had been soaked in ninhydrin stain. Another non-thermophilic bacterial $(+)-\gamma$-lactamase that can also carry out this reaction has been identified within the bacterial Delftia species (PDB code 2WKN, Gonsalvez et al., 2001). This enzyme is of a different class, structure, and mechanism from the archaeal enzyme but both can use the non-natural $\gamma$-lactam as a substrate. It is related to the bi-zinc containing metalloprotease formamidase by analysis using the SCOP2 database (Andreeva et al., 2014). A third (-)- $\gamma$-lactamase of opposite stereoselectivity is related to a $\alpha / \beta$ hydrolase fold esterase enzyme which can also carry out the side reaction of bromination at $\mathrm{pH} 4.0$ and was referred to as a non-cofactor containing bromoperoxidase (Line et al., 2004).
The Sulfolobus (+)- $\boldsymbol{\gamma}$-lactamase has been cloned and overexpressed in Escherichia coli and purified to homogeneity (Toogood et al., 2004). The molecular mass of the monomer was estimated to be $55 \mathrm{kDa}$ by SDS-PAGE, which is consistent with the calculated molecular mass of $55.7 \mathrm{kDa}$. The native molecular weight was $110 \mathrm{kDa}$ as determined by gel filtration chromatography, indicating that the enzyme exists as a dimer in solution. The purified enzyme has been crystallized with a view to determining its three-dimensional structure.

This thermostable archaeal (+)- $\boldsymbol{\gamma}$-lactamase has a high sequence homology to the signature amidase family of enzymes. It shows similar inhibition to the amidase enzymes with benzonitrile, phenylmethylsulfonyl fluoride, and heavy metals such as mercury, and it is activated by thiol reagents. Alignment of the amino acid sequences of the $(+)-\gamma$-lactamase from $S$. solfataricus with four amidases from Pseudomonas chlororaphis B23, Rhodococcus sp. N-771, Rhodococcus erythropolis N-774, and Rhodococcus rhodochrous J shows it has a $41-44 \%$ sequence identity to these enzymes. The amidases belong to the signature amidase family as they all contain the consensus sequence GGSS(S/G)GS. The amino acid sequence of the $\gamma$-lactamase contains the highly conserved putative catalytic residues aspartic acid and serine but not the highly conserved cysteine residue (Kobayashi et al., 1997).

The purified $(+)-\gamma$-lactamase enzyme has been immobilized as a cross-linked, polymerized enzyme preparation and packed into microreactors (Hickey et al., 2009). The thermophilic $(+)-\gamma$-lactamase retained $100 \%$ of its initial activity at the assay temperature, $80^{\circ} \mathrm{C}$, for $6 \mathrm{~h}$ and retained $52 \%$ activity after $10 \mathrm{~h}$, indicating the advantage of the immobilization. The free enzyme began to display a reduction in activity after $1 \mathrm{~h}$ at $80^{\circ} \mathrm{C}$. The higher stability of the immobilized enzyme provided the advantage that it could be used to screen many compounds in a microreactor system without denaturation.

\section{L-Aminoacylase and Pyroglutamyl Carboxyl Peptidase from the Thermophilic Archaeon Thermococcus litoralis}

Many pharmaceutically active compounds contain nitrogen and can be derived from amino acids (Drauz, 1997). These compounds need to be optically pure, which can be achieved by the use of specific L or D aminoacylase enzymes. There is a large growth in the use of unnatural amino acids, for example, L-tert-leucine is a precursor to many pharmaceutically active compounds such as the antitumor compounds (Bommarius et al., 1998). A thermophilic archaeal L-aminoacylase has been cloned and overexpressed from the archaeon Thermococcus litoralis (Toogood et al., 2002a). The enzyme was identified from a Thermococcus DNA expression library which gave a positive hit for esterase activity. This esterase gene was later found to code for a pyroglutamyl carboxyl peptidase, which is a novel cysteine protease that cleaves the pyroglutamyl group from the $\mathrm{N}$-terminus of biologically important peptides. The enzyme has been characterized both biochemically and structurally and demonstrated to be a new class of cysteine protease (Singleton et al., 1999). The commercial use of this enzyme is to cleave the 
pyroglutamyl group from "blocked" peptides allowing them to be N-terminally sequenced. The Thermococcus protease forms a tetrameric structure held together by disulfide bonds between the dimer subunit interface and is stabilized by an unusual hydrophobic core at the center of the tetrameric structure. This unusual feature is formed from four two-stranded antiparallel $\beta$-sheets, one from each subunit. The sheets are built from the hydrophobic amino acid residues Phe-Phe-Leu-Leu (Figure 1). This hydrophobic insertion is unique to the T. litoralis enzyme, with no equivalent structure seen in other bacterial or archaeal pyroglutamyl carboxyl peptidase enzymes (Singleton et al., 1999).

The Thermococcus L-aminoacylase enzyme which was identified next to the novel cysteine protease and has an $82 \%$ sequence identity to an L-aminoacylase from Pyrococcus furiosus (Tanimoto et al., 2008) and 45\% sequence identity to a carboxypeptidase from another thermophilic archaeon S. solfataricus. The Thermococcus enzyme is not inhibited by conventional aminoacylase inhibitors such as mono-tert-butyl malonate so appears to be novel. It is thermostable, with a half-life of $25 \mathrm{~h}$ at $70^{\circ} \mathrm{C}$ and has an optimal activity at $85^{\circ} \mathrm{C}$ in Tris- $\mathrm{HCl}$, $\mathrm{pH}$ 8.0. This $\mathrm{T}$. litoralis L-aminoacylase has a broad substrate specificity preferring the amino acids: Phe $\gg$ Met $>$ Cys $>$ Ala $\simeq$ Val $>$ Tyr $>$ Propargylglycine $>$ Trp $>$ Pro $>$ Arg. A column bioreactor containing the recombinant Thermococcus enzyme has been constructed by immobilization onto Sepharose beads. This bioreactor showed no loss of activity towards the substrate $N$-acetyl-DL-Trp after 5 days and $32 \%$ of the activity remained after 40 days at $60^{\circ} \mathrm{C}$ (Toogood et al., 2002b). The enzyme has more recently been immobilized by covalent attachment to an epoxy resin formed in channels of a microreactor allowing a "flow-through" procedure to be used (Ngamsom et al., 2010). This

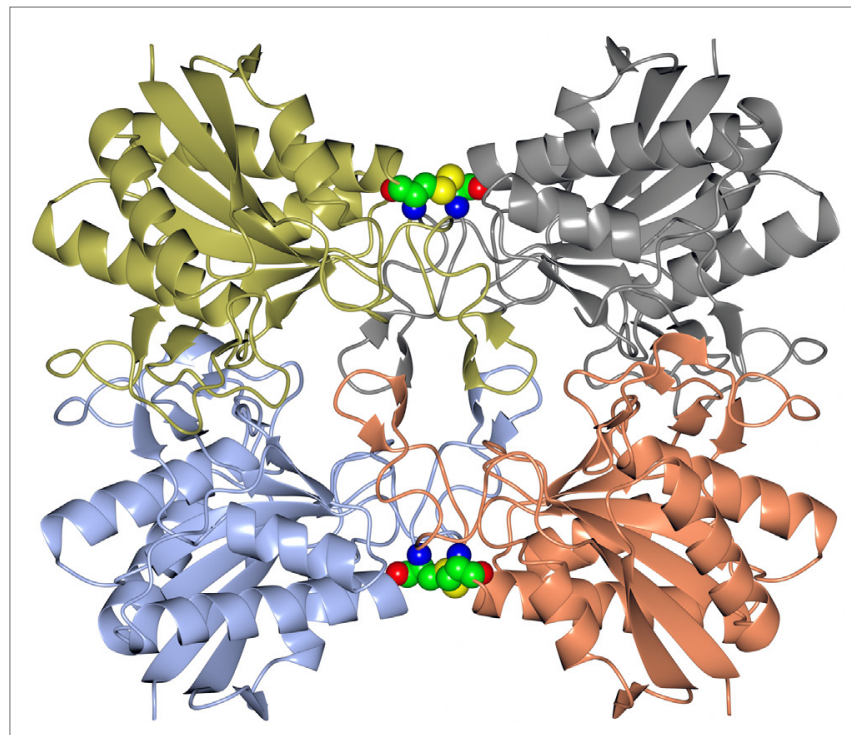

FIGURE 1 | A diagram showing the tetrameric structure of the Thermococcus pyroglutamyl carboxyl peptidase. The secondary structural elements are shown in a different color for each subunit. The hydrophobic core structure is shown in the center of the molecule and the disulfide bonds at the subunit interfaces in sphere mode (Singleton et al., 1999, PDB code 1A2Z). can be used for rapid substrate screening of the L-aminoacylase and eliminates potential substrate and product inhibition. It has been shown in pilot-scale biotransformation reactions using the substrate $\mathrm{N}$-acetyl-DL-propargylglycine that this enzyme does show substrate inhibition (Toogood et al., 2002a).

The Thermococcus L-aminoacylase enzyme is now being used for multiton commercial production of $\mathrm{L}$-amino acids and their analogs by Chirotech/Dow Pharma and more recently by Chirotech/Dr Reddys (Holt, 2004). A racemase enzyme has been developed in order to convert the isomer not used by the enzyme to the form that is used which can enable a more efficient process resulting in a $100 \%$ conversion of the racemic substrate (Baxter et al., 2012).

There are differences in substrate specificity between the Thermococcus L-aminoacylase and another thermophilic archaeal enzyme from Pyrococcus species. The substrate $\mathrm{N}$-acetyl-L-phenylalanine is the most favorable substrate for the Thermococcus enzyme; however, this substrate is not used by the Pyrococcus L-aminoacylase (Tanimoto et al., 2008).

\section{Carboxyl Esterase from a Thermophilic Bacterium Thermogutta terrifontis}

Esterases are a class of commonly used enzymes in industrial applications. This is partially due to their inherent stability in organic solvents and the ability to freely reverse the enzyme reaction from hydrolysis to synthesis by the elimination of the water that is used during the hydrolysis mechanism. The carboxyl esterases catalyze the hydrolysis of the ester bond of relatively small water-soluble substrates. A new carboxyl esterase (TtEst) has been identified in a recently identified thermophilic bacterium Thermogutta terrifontis from the phylum Planctomycetes. This enzyme has been cloned and overexpressed in E. coli (Sayer et al., 2015). The enzyme has been characterized biochemically and shown to have activity towards small $p$-nitrophenyl $(p N P)$ carboxylic esters with optimal activity for $p$ NP-propionate. The TtEst enzyme is very thermostable and retained $95 \%$ of its activity after incubation for $1 \mathrm{~h}$ at $80^{\circ} \mathrm{C}$. The enzyme has been crystallized and its structure determined without ligands bound in the active site and in complex with a substrate analog D-malate and the product acetate. The bound ligands in the structure have allowed the identification of the carboxyl and alcohol binding pockets in the enzyme active site (Figure 2). It has also allowed a detailed comparison with structurally related enzymes that has given insight into how differences in the catalytic activity can be rationalized based on the properties of the amino acid residues in different active site pockets. An overall comparison of the alcohol binding pocket in TtEst with the equivalent pocket in the 3-oxoadipate-enol lactonase from Burkholderia xenovorans (PcaD) with 29\% sequence identity (PDB code 2XUA) (Bains et al., 2011) and Aureobacterium species (-) $\gamma$-lactamase (Agl) with $30 \%$ sequence identity (PDB code $1 \mathrm{HKH}$ ) (Line et al., 2004) has been carried out. The catalytic triad residues and the position of the oxyanion hole are conserved between these enzymes. The PcaD and Agl show that the TtEst pocket has a much more polar and charged environment in the active site, which allows the binding of organic acids such as D-malate where the distant carboxyl is coordinated by Arg139 and Tyr105. The PcaD and Agl 


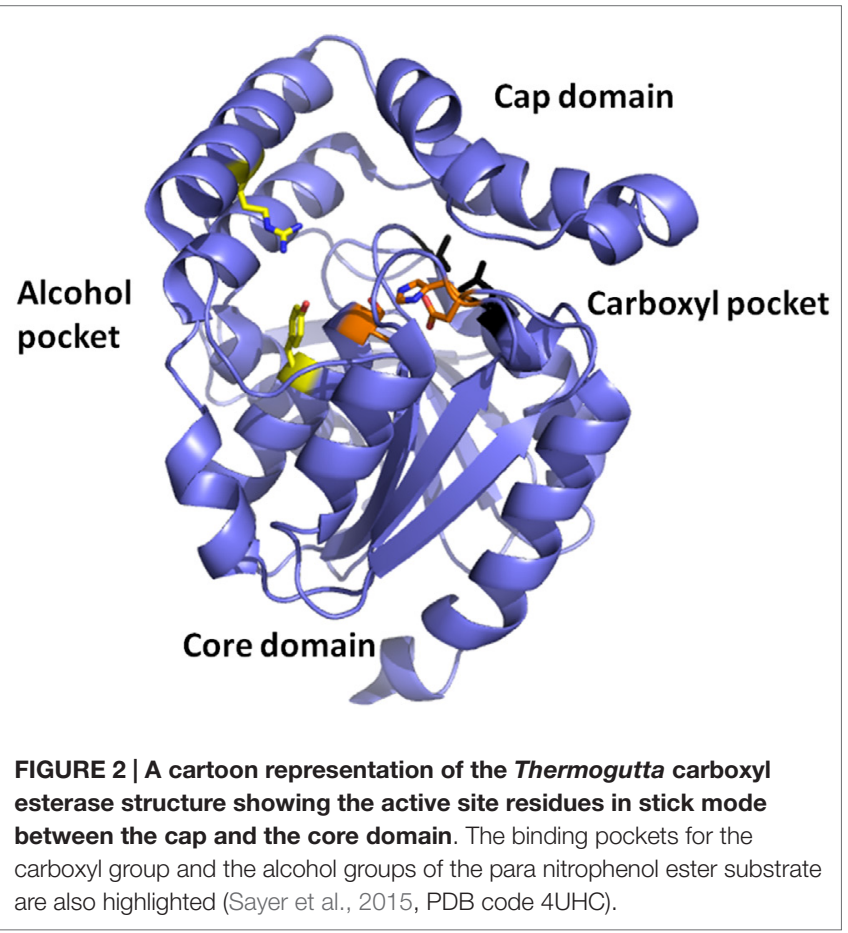

enzymes have more hydrophobic substrate binding pockets, with the residues Arg139 and Tyr105 in TtEst replaced by Trp135 and Ile129 in PcaD and Trp204 and Leu125 in Agl. In both PcaD and Agl, the active site is better suited for the binding of structures such as lactone and $\gamma$-lactam rings. Similarly, the Pseudomonas fluorescens esterase with $30 \%$ sequence identity (PDB code $3 \mathrm{HEA}$ ) (Yin et al., 2010) has an alcohol pocket where the structure is lined with several hydrophobic phenylalanine side chains that should have affinity for the lactone ring. This would explain its lactonase activity towards caprolactone (Cheeseman et al., 2004) in addition to the esterase activity.

Mutant enzymes have been constructed to extend the substrate range of T. terrifontis esterase to accept the larger butyrate and valerate $p \mathrm{NP}$ esters. These mutant enzymes have also shown a significant increase in activity towards acetate and propionate $p \mathrm{NP}$ esters. A crystal structure of the Leu37Ala mutant has been determined with the butyrate product bound in the carboxyl pocket of the active site. The mutant structure shows an expansion of the pocket that binds the substrate carboxyl group, which is consistent with the observed increase in activity towards $p$ NP-butyrate.

\section{A $\alpha$-Carbonic Anhydrase from the Thermophilic Bacterium Thermovibrio ammonificans}

Carbonic anhydrase enzymes catalyze the reversible hydration of carbon dioxide to bicarbonate. A stable robust $\alpha$-carbonic anhydrase has been identified in the thermophilic bacterium Thermovibrio ammonificans. The enzyme has been cloned and overexpressed in E. coli. This protein has been characterized both biochemically and structurally (James et al., 2014). The crystal

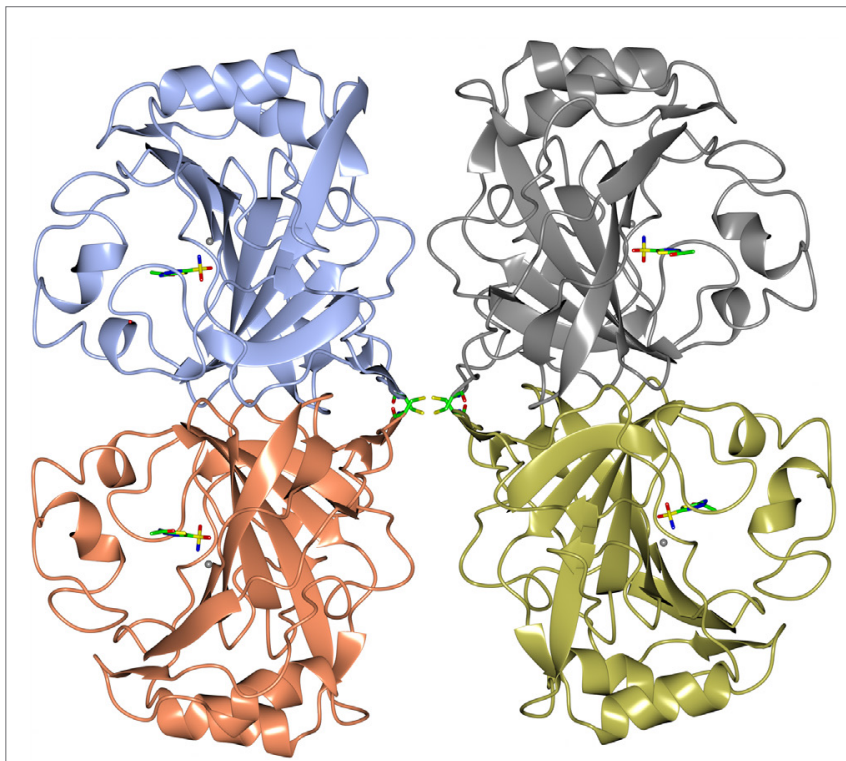

FIGURE 3 | A diagram of the thermophilic carbonic anhydrase from Thermovibrio showing the tetrameric structure held together by disulfide bonds in the center of the molecule (James et al., 2014 PDB code 4UOV).

structure of this enzyme has been determined in its native form and in two complexes with bound inhibitors. It is unusual since it forms a tetrameric structure rather than the dimer reported for some previously studied related enzymes. The Thermovibrio enzyme is stabilized by a unique core in the center of the molecule formed by two intersubunit disulfide bonds and a single lysine residue from each monomer (Figures 3 and 4). The structure of this central core region protects the intersubunit disulfide bonds from reduction. The enzyme is located in the endoplasmic reticulum of Thermovibrio as evidenced by the presence of an $\mathrm{N}$-terminal signal peptide. When the recombinant protein is oxidized to mimic the natural environment of the periplasmic space, it shows an increase in thermostability and retains $90 \%$ of its activity after incubation at $70^{\circ} \mathrm{C}$ for $1 \mathrm{~h}$. These properties make it a good candidate for commercial carbon dioxide capture. Another thermophilic bacterial $\alpha$-carbonic anhydrase has been described from Sulfurihydrogenibium yellowstonense. This carbonic anhydrase is also thermostable and is a dimer stabilized by ionic networks (Di Fiore et al., 2013).

The thermophilic bacteria appear to contain the high-activity $\alpha$-carbonic anhydrase enzymes that have a similar structure to the well-studied bovine carbonic anhydrase enzyme, whereas the archaea have carbonic anhydrases that have different structures and mechanisms. There are six distinct families of CAs $(\alpha, \beta, \gamma$, $\delta$, $\zeta$, and $\eta$ ) (Smith et al., 1999; Guler et al., 2015). The amino acid sequences are conserved between each family; however, there is no sequence or structural similarity between the different families. The carbonic anhydrase activity requires the presence of a catalytic zinc ion which is coordinated to either histidine or cysteine amino acids depending on the class of the enzyme (Silverman and Lindskog, 1988). 


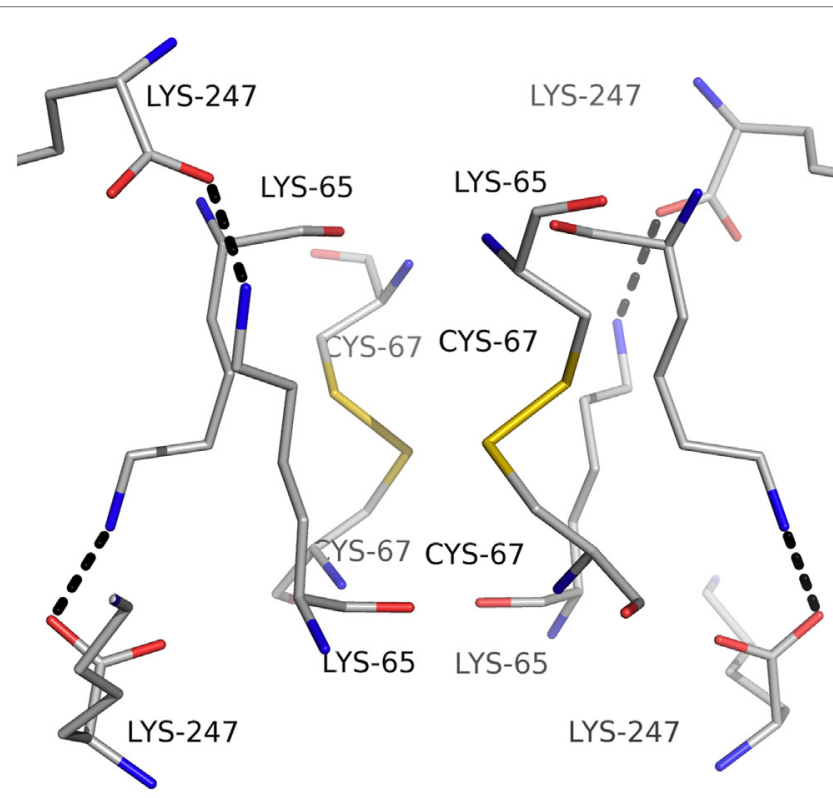

FIGURE 4 | A diagram showing the unusual structural feature of disulfide bonds in the center of the Thermovibrio carbonic anhydrase which are shielded by lysine amino acid residues (James et al., 2014, PDB code 4UOV)

\section{A Thermophilic Transaminase Enzyme from Sulfolobus solfataricus}

The transaminase enzymes are important biocatalysts for the pharmaceutical industries since they produce chiral amines which are components of a range of different drug molecules. The archaeon S. solfataricus has been found to be an interesting source of a thermostable transaminase enzyme of the group IV Pfam group (Sayer et al., 2012). This pyridoxal phosphate (PLP)-containing enzyme is involved in the non-phosphorylated pathway for serine synthesis which is not found in bacteria but is found in animals and plants. The Sulfolobus transaminase carries out the conversion of L-serine and pyruvate to 3-hydroxypyruvate and alanine. It also has activity towards methionine, asparagine, glutamine, phenylalanine, histidine, and tryptophan and can be used in a cascade reaction with a $\mathrm{C}-\mathrm{C}$ bond making enzyme, transketolase, for the synthesis of optically pure drug intermediates (Chen et al., 2006).

The dimeric thermophilic archaeal transaminase enzyme structure has been solved in the holo form of the enzyme and in complex with an inhibitor gabaculine and in a substrate complex with phenolpyruvate, the keto product of phenylalanine (Sayer et al., 2012). Figure 5 shows a cartoon diagram of the dimeric $S$. solfataricus transaminase with an inhibitor bound to the cofactor PLP in the two active sites. The structural studies of this enzyme have given some insight into the conformational changes around the active site occurring during catalysis and have helped to understand the enzyme's substrate specificity (Sayer et al., 2012). How different members of the PLP enzyme family are able to accept a variety of substrates is vitally important to understand for the use of these enzymes in commercial applications.

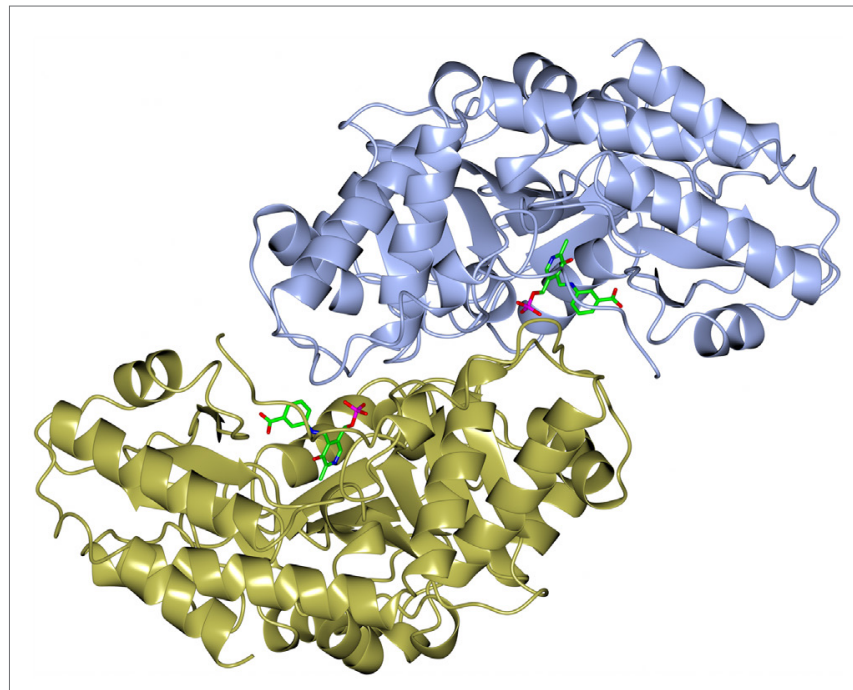

FIGURE 5 | A diagram of the structure of the Sulfolobus transaminase dimer showing the cofactor pyridoxal phosphate (PLP) forming an irreversible complex with the inhibitor gabaculine, shown in stick mode in the two active sites (Sayer et al., 2012, PDB code 3ZRP).

The Sulfolobus transaminase is relatively thermostable for $10 \mathrm{~min}$ at $70^{\circ} \mathrm{C}$ and at $\mathrm{pH}$ 6.5. Features of the archaeal enzyme that relate to its increased stability when compared with a mesophilic related yeast enzyme show that the Sulfolobus enzyme has 21 salt bridges compared to 10 in the mesophilic enzyme including several three to four amino acid networks which offer increased stability. There is a C-terminal extension in the Sulfolobus enzyme and shorter surface loops which are all general features that are found in thermophilic enzymes. The Sulfolobus transaminase dimer interface is unusual being hydrophobic in nature with few ionic interactions which are generally associated with more thermophilic archaeal enzymes. This Sulfolobus serine transaminase is the first example of a thermophilic archaeal serine transaminase to be studied structurally and is shown to have properties that meet the requirements for the commercial application of the enzyme in biocatalysis.

\section{New Epoxide Hydrolases from Extremophilic Metagenomes}

An important enzyme activity of interest to the pharmaceutical industry is the ability to catalyze the hydrolysis of an oxirane (epoxide) ring by addition of a molecule of water to form a vicinal diol as a product (Widersten et al., 2010; Kotik et al., 2012). The enzymes that can carry out this reaction are ubiquitously expressed in all living organisms and they play an important physiological role in the detoxification of reactive xenobiotics or endogenous metabolites and in the formation of biologically active mediators. The epoxide hydrolases are already used for the production of optically pure epoxides and diols which are important synthons for the preparation of fine chemicals and drugs, for example, the chiral precursors of $\beta$-blockers (Kong et al., 2014; Nestl et al., 2014). The epoxide hydrolase enzymes fall into two classes with completely different $3 \mathrm{D}$ structures, the $\alpha / \beta$ hydrolase 


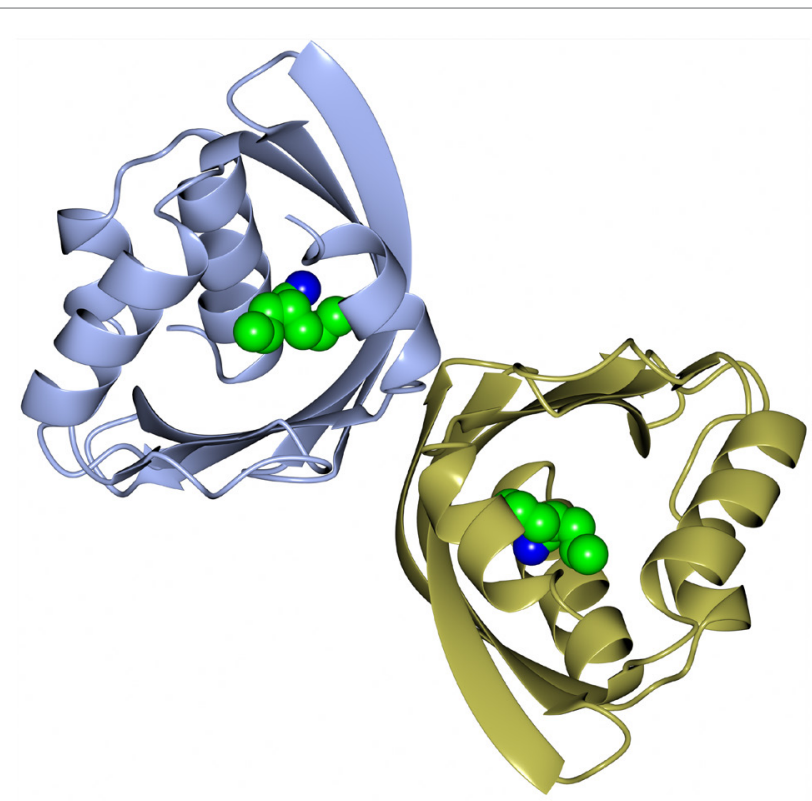

FIGURE 6 | A diagram showing the thermophilic limonene epoxide hydrolase isolated from the metagenomic sample collected from hot springs in Russia. The inhibitor, valpromide, is bound into the active site and is shown in sphere mode (Ferrandi et al., 2015a, PDB code 5AlH).

fold class and the limonene class (LEH) of which only few have been fully characterized. The LEH enzyme active site contains three residues (Asp, Arg, and Asp) that have been proposed to act in a concerted fashion to activate a water molecule which is able to open the epoxide ring without the formation of a covalently bound alkyl-enzyme intermediate (Arand et al., 2003; Hopmann et al., 2005).

Recently, as part of a thermophilic metagenomic project, two new thermostable epoxide hydrolases of the limonene class have been discovered. The metagenomes were isolated in Russia and China from hot terrestrial environments growing at $46^{\circ} \mathrm{C}$ and $55^{\circ} \mathrm{C}$ and at neutral $\mathrm{pH}$. A bioinformatic approach was used to identify the genes coding for these industrially important enzymes which have been cloned and overexpressed in E. coli. The resultant proteins have been fully characterized as far as their biochemical properties, specificity, stereoselectivity, and crystal structure (Ferrandi et al., 2015a). The structure of the LEH from the Russian metagenome is shown in Figure 6. The new LEH enzymes have also been further evaluated and used in pilot-scale biotransformations for industrial applications (Ferrandi et al., 2015b).

\section{DEHALOGENASE ENZYMES FROM EXTREMOPHILIC BACTERIA AND ARCHAEA}

\section{L-Haloacid Dehalogenase from the Thermophilic Archaeon Sulfolobus tokadii}

A thermophilic dehalogenase enzyme of industrial interest is found in the archaeon Sulfolobus tokadaii. This L-haloacid dehalogenase enzyme has been cloned and overexpressed in $E$. coli and has been characterized biochemically and structurally (Rye et al., 2007, 2009). This enzyme has applications for chiral halo-carboxylic acid production and bioremediation. Chiral halocarboxylic acids are important intermediates in the fine chemical/ pharmaceutical industries. The Sulfolobus dehalogenase enzyme has the potential to resolve racemic mixtures of bromocarboxylic acids and is able to catalyze the conversion of 2-halo-carboxylic acids to the corresponding hydroxyalkanoic acids. It has been shown to display activity towards longer chain substrates than the bacterial Xanthomonas autotrophicus dehalogenase (Van der Ploeg et al., 1991) with activity seen towards 2-chlorobutyric acid due to a more accessible active site (Rye et al., 2009). The enzyme has a maximum activity at $60^{\circ} \mathrm{C}$ and a half-life of over an hour at $70^{\circ} \mathrm{C}$. It is stabilized by a salt bridge and hydrophobic interactions on the subunit interface, helix capping, a more compact subdomain than related enzymes, and shortening of surface loops. A related hyperthermophilic Pyrococcus dehalogenase (29\% sequence identity) whose structure is available from a structural genomics project is a monomeric enzyme stabilized by a disulfide bond (Arai et al., 2006).

\section{Dehalogenases from the Marine Environment}

The marine environment has been recognized as a potential source of novel enzymes (Trincone, 2011). A novel L-haloalkane dehalogenase has been biochemically and structurally characterized from the psychrophilic bacterium Psychromonas ingrahamii (Novak et al., 2013a). This organism was originally isolated in 1991 from Elson Lagoon, Point Barrow Alaska. Original samples were collected from the sea ice interface, where temperatures can reach $-10^{\circ} \mathrm{C}$ (Breezee et al., 2004). The P. ingrahamii is a nonmotile, large, rod-shaped bacterium that can utilize glycerol as a sole carbon source. The $P$. ingrahamii genome has been shown to encode numerous genes that synthesize polysaccharides and betaine choline. These compounds are thought to aid survival of the organism at low temperatures (Riley et al., 2008). Studies into the structural properties of proteins from psychrophilic organisms have shown they have enhanced structural flexibility at low temperatures (Feller, 2003; Feller and Gerday, 2003). The fine balance between activity, stability, and flexibility of proteins which control enzyme kinetics has been reviewed by Georlette et al. (2004). When proteins from psychrophiles are compared with the equivalent proteins from mesophiles, they show a decrease in the number of ionic interactions, decreased number of hydrogen bonds, and fewer hydrophobic amino acid residues. The L-haloacid dehalogenase from $P$. ingrahamii has been cloned and overexpressed in E. coli. The recombinant protein has been biochemically and structurally characterized and compared with mesophilic and thermophilic L-haloacid dehalogenases. It shows activity towards monobromoacetic (100\%), monochloroacetic acid (62\%), S-chloropropionic acid (42\%), S-bromopropionic acid (31\%), dichloroacetic acid (28\%), and 2-chlorobutyric acid (10\%). The L-haloacid dehalogenase has highest activity towards substrates with shorter carbon chain lengths $(\leq \mathrm{C} 3)$, without preference towards a chlorine or bromine at the $\alpha$-carbon position. 
The enzyme has an optimal temperature for activity at $45^{\circ} \mathrm{C}$ and retains $70 \%$ of its activity after being incubated at $65^{\circ} \mathrm{C}$ for $90 \mathrm{~min}$. The enzyme is relatively stable in organic solvents and therefore shows mesophilic properties despite being isolated from a psychrophilic bacterium. The relatively high thermal stability and optimal temperature for activity is surprising for an enzyme isolated from a psychrophilic bacterium. The thermal stability results show that the enzyme is stable beyond its catalytic temperature optimum. The "equilibrium model" has previously been described to explain the difference between apparent temperature optimum and higher thermostability for a range of psychrophilic, mesophilic, and thermophilic enzymes (Lee et al., 2007). This model includes an inactivated state of the enzyme at temperatures above the optimally active form, which are in reversible equilibrium. At sufficiently higher temperatures, the folded but inactive form of the enzyme can undergo irreversible thermal inactivation to the denatured state (Daniel and Danson, 2010).

Many reported psychrophilic enzymes have highest catalytic efficiency at low temperatures and have low thermal stability. However, some psychrophilic enzymes have been shown to denature at higher temperatures than that they appear to be inactive. For example, the Pseudoalteromonas haloplanktis DNA ligase is optimally active below $20^{\circ} \mathrm{C}$, inactive above $25^{\circ} \mathrm{C}$, but is not fully denatured until $35^{\circ} \mathrm{C}$ (Georlette et al., 2003). Also the citrate synthase from an Antarctic bacterium has been shown to decrease in enzyme activity at temperatures above its temperature optimum; however, this is not due to thermal denaturation of the enzyme since the activity loss can be reversed as the temperature decreases (Gerike et al., 1997, 2001). Further studies on psychrophilic enzymes will help to understand how these enzymes are adapted to function at low temperatures.

An homology model of the marine L-haloacid dehalogenase has been built based on the crystal structure of related enzymes. The active site pocket of the $P$. ingrahamii model and the $S$. tokodaii and Burkholderia cepacia enzymes are highly similar, with almost all residues in similar conformations. The observed thermostability of the enzyme is consistent with the conclusions drawn from homology modeling where no obvious psychrophilic adaptations were observed. At the in vitro optimal growth temperature of $P$. ingrahamii, L-haloacid dehalogenase would not be active. This could indicate that the enzyme has been acquired by horizontal gene transfer. This solvent-resistant and stable L-haloacid dehalogenase from $P$. ingrahamii has potential to be used as a biocatalyst in industrial processes.

Another novel marine dehalogenase from the Rhodobacteraceae family has been isolated from a polychaeta worm collected from Tralee beach, Argyll, UK. The enzyme tested positive for L-haloacid dehalogenase activity towards L-monochloropropionic acid (Novak et al., 2013b). A diagram of the overall two-domain structure is shown in Figure 7 with the active site aspartic acid highlighted in the cleft between the domains. The active site of this dehalogenase shows significant differences from previously studied L-haloacid dehalogenases. The asparagine and arginine residues shown to be essential for catalytic activity in other L-haloacid dehalogenases are not present in this enzyme. The histidine residue that replaces the asparagine residue as shown in the structure was coordinated

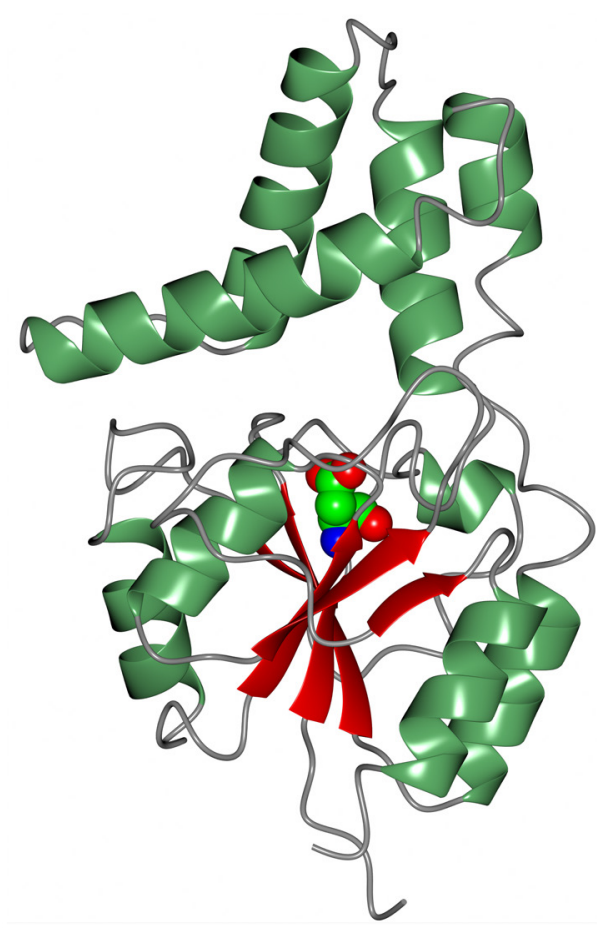

FIGURE 7 | A diagram of the Rhodobacteraceae L-haloacid dehalogenase showing the smaller cap domain at the top of the molecule and the Rossmann-like fold domain at the bottom. The catalytic aspartic acid is shown in sphere mode at the interface between the two domains (Novak et al., 2013b, PDB code 2YML).

by a conformationally strained glutamate residue that replaces a conserved glycine residue. The His/Glu dyad is positioned for deprotonation of the catalytic water which attacks the ester bond in the reaction intermediate. The catalytic water in this novel enzyme is shifted by from its position in other L-haloacid dehalogenases. A similar His/Glu or Asp dyad is known to activate the catalytic water in haloalkane dehalogenases. The novel enzyme represents a new member within the L-haloacid dehalogenase family and appears to have evolved with properties of a mixture of a haloalkane dehalogenase and a haloacid dehalogenase, and it has the potential to be used as a commercial biocatalyst. It is not unusual to find such novel enzymes in extremophilic microorganisms.

The use of psychrophilic enzymes in industrial processes allows instability issues with reactants and products to be avoided and results in a reduction in cost because of the lower energy consumption. High catalytic efficiency at low temperatures makes psychrophilic enzymes attractive for use in biocatalytic processes (Gomes and Steiner, 2004; Novak and Littlechild, 2013).

\section{CONCLUSION}

Extremophilic enzymes are becoming an important source of new industrially robust biocatalysts. The use of nature's biodiversity provides an ever increasing resource of new genomes and metagenomes to identify useful activities which can carry out a variety of 
chemical biotransformations of commercial interest. The activity of the enzymes can be identified by both bioinformatic techniques and screening of expression libraries. The enzymes can be cloned and overexpressed in easily grown hosts such as E. coli allowing access to sufficient quantities of the purified enzymes for detailed biochemical and structural characterization. The scale-up of the enzyme production required for commercial applications can be carried out by using a fungal host system that allows export of the proteins into the growth medium for easy downstream processing, if appropriate. The cost of the enzyme biocatalyst must be matched to the value of the end product. Higher value optically pure pharmaceutical intermediates which are used as building blocks for drug intermediates will allow a higher enzyme price than enzymes used for the production of bulk chemicals, additives for domestic products, food production, or biomass degradation processes. The stability of the biocatalyst is also an economic issue since if the enzyme is sufficiently robust under the industrial conditions it can be used for repeated cycles of the biocatalytic process thereby saving money. The use of enzymes isolated from extremophilic microorganisms offers the opportunity to access enzymes that are stable in a variety of different conditions such as high temperatures, low temperatures, high salt concentrations, high pressure, extremes of $\mathrm{pH}$, and often a combination of these properties, which can make them more suited to the industrial environments.

The use of enzymes in "white biotechnology" is expected to grow with biobased materials and chemicals from emerging technologies predicted to rise globally to over 7.4 million metric tons in 2018 (Lux Research Analysis, www.luxresearchinc.com). Each

\section{REFERENCES}

Andreeva, A., Howorth, D., Chothia, C., Kulesha, E., and Murzin, A. G. (2014). SCOP2 prototype: a new approach to protein structure mining. Nucleic Acids Res. 42, D310-D314. doi:10.1093/nar/gkt1242

Arai, R., Kukimoto-Niino, M., Kuroishi, C., Bessho, Y., Shirouzu, M., and Yokoyama, S. (2006). Crystal structure of the probable haloacid dehalogenase PH0459 from Pyrococcus horikoshii OT3. Protein Sci. 15, 373-377. doi:10.1110/ps.051922406

Arand, M., Hallberg, B. M., Zou, J., Bergfors, T., Oesch, F., van der Werf, M. J., et al. (2003). Structure of Rhodococcus erythropolis limonene-1,2-epoxide hydrolase reveals a novel active site. EMBO J. 22, 2583-2592. doi:10.1093/emboj/cdg275

Bains, J., Kaufman, L., Farnell, B., and Boulanger, M. J. (2011). A product analog bound form of 3-oxoadipate-enol-lactonase $(\mathrm{PcaD})$ reveals a multifunctional role for the divergent cap domain. J. Mol. Biol. 406, 649-658. doi:10.1016/j. jmb.2011.01.007

Baxter, S., Royer, S., Grogan, G., Brown, F., Holt-Tiffin, K., Taylor, I., et al. (2012). An improved racemase/acylase biotransformation for the amino acids. J. Am. Chem. Soc. 134, 19310-19313. doi:10.1021/ja305438y

Bommarius, A. S., Schwarm, M., and Drauz, K. (1998). Biocatalysis to aminoacid-based chiral pharmaceuticals: examples and perspectives. J. Mol. Catal. B Enzym. 5, 1-11. doi:10.1016/S1381-1177(98)00009-5

Breezee, J., Cady, N., and Staley, J. T. (2004). Subfreezing growth of the sea ice bacterium Psychromonas ingrahamii. Microb. Ecol. 47, 300-304. doi:10.1007/ s00248-003-1040-9

Cheeseman, J. D., Tocilj, A., Park, S., Schrag, J. D., and Kazlauskas, R. J. (2004). Structure of an aryl esterase from Pseudomonas fluorescens. Acta Crystallogr. D60, 1237-1243. doi:10.1107/S0907444904010522

Chen, B. H., Sayar, A., Kaulmann, U., Dalby, P. A., Ward, J. M., and Woodley, J. M. (2006). Reaction modelling and simulation to assess the integrated use of transketolase and $\omega$-transaminase for the synthesis of an aminotriol. Biocatal. Biotransformation 24, 449-457. doi:10.1080/10242420601068668 industrial process is different and the correct biocatalyst needs to be identified and optimized for the industrial application. Many enzyme families have not realized their potential in this area and remain to be discovered. Enzymes that can catalyze reactions with non-natural substrates and under non-physiological conditions, which are often used in industry, can be found in the extremophile environment. Although the techniques available for enzyme engineering have improved recently, the enzyme discovery and optimization process is still a limiting factor for the adoption of new biobased industrial processes.

\section{ACKNOWLEDGMENTS}

The author would like to thank the University of Exeter, the Wellcome Trust, the BBSRC, the EPSRC, and the Technology Strategy Board, UK, for sponsoring research in the JL Laboratories at the Exeter Biocatalysis Centre. The EU Framework 7 grant "HotZyme" entitled Systematic Screening of Organisms from Hot Environments, Grant Number 265933, is thanked for supporting studies on the isolation of the metagenomes from Russia and China where the novel thermophilic limonene epoxide hydrolases were isolated and for the identification of the first thermophilic Planctomycetes species where the novel thermophilic esterase was identified. This EU project sponsored the research to clone, overexpress, and biochemically and structurally characterize these three new enzymes to allow their potential commercial exploitation. JL would like to thank all of the collaborators, postdoctoral fellows, and students who have contributed to the research work covered in this paper.

Daniel, R. M., and Danson, M. J. (2010). A new understanding of how temperature affects the catalytic activity of enzymes. Trends Biochem. Sci. 35, 584-591. doi:10.1016/j.tibs.2010.05.001

Di Fiore, A., Capasso, C., De Luca, V., Monti, S. M., Carginale, V., Supuran, C. T., et al. (2013). X-ray structure of the first 'extremo- $\alpha$-carbonic anhydrase', a dimeric enzyme from the thermophilic bacterium Sulfurihydrogenibium yellowstonense YO3AOP1. Acta Crystallogr. D69, 1150-1159. doi:10.1107/ S0907444913007208

Drauz, K. (1997). Chiral amino acids: a versatile tool in the synthesis of pharmaceuticals and fine chemicals. Int J Chem Chimia 51, 310-314.

Feller, G. (2003). Molecular adaptations to cold in psychrophilic enzymes. Cell. Mol. Life Sci. 60, 648-662. doi:10.1007/s00018-003-2155-3

Feller, G., and Gerday, C. (2003). Psychrophilic enzymes: hot topics in cold adaption. Nat. Rev. Microbiol. 1, 200-208. doi:10.1038/nrmicro773

Ferrandi, E. E., Sayer, C., Isupov, M. N., Annovazzi, C., Marchesi, C., Iacobone, G., et al. (2015a). Discovery and characterization of thermophilic limonene-1,2-epoxide hydrolases from hot spring metagenomics libraries. FEBS J. 282, 2879-2894. doi:10.1111/febs.13328

Ferrandi, E., Marchesi, C., Annovazzi, C., Riva, S., Monti, D., and Wohlgemuth, R. (2015b). Efficient epoxide hydrolase-catalyzed resolutions of (+)- and (-)-cis/trans-limonene oxides. ChemCatChem doi:10.1002/cctc.201500608

Georlette, D., Blaise, V., Collins, T., D’Amico, S., Gratia, E., Hoyoux, A., et al. (2004). Some like it cold: biocatalysis at low temperatures. FEMS Microbiol. Rev. 28, 25-42. doi:10.1016/j.femsre.2003.07.003

Georlette, D., Damiens, B., Blaise, V., Depiereux, E., Uversky, V. N., Gerday, C., et al. (2003). Structural and functional adaptations to extreme temperatures in psychrophilic, mesophilic and thermophilic DNA ligases. J. Biol. Chem. 278, 37015-37023. doi:10.1074/jbc.M305142200

Gerike, U., Danson, M. J., and Hough, D. W. (2001). Cold-active citrate synthase: mutagenesis of active-site residues. Protein Eng. 14, 655-661. doi:10.1093/ protein/14.9.655 
Gerike, U., Danson, M. J., Russell, N. J., and Hough, D. W. (1997). Sequencing and expression of the gene encoding a cold active citrate synthase from an Antarctic bacterium, strain DS2 3R. Eur. J. Biochem. 248, 49-57. doi:10.1111/j.1432-1033.1997.00049.x

Gomes, J., and Steiner, W. (2004). The biocatalytic potential of Extremophiles and Extremozymes. Food Technol. Biotechnol. 42, 223-235.

Gonsalvez, I. S., Isupov, M., and Littlechild, J. A. (2001). Crystallization and preliminary X-ray analysis of a gamma-lactamase. Acta Crystallogr. D57, 284-286. doi:10.1107/S0907444900016838

Guler, O. O., Capasso, C., and Supuran, C. T. (2015). A magnificent enzyme superfamily: carbonic anhydrases, their purification and characterization. J. Enzyme Inhib. Med. Chem. doi:10.3109/14756366.2015.1059333

Hickey, A. M., Ngamsom, B., Wiles, C., Greenway, G. M., Watts, P., and Littlechild, J. A. (2009). A microreactor for the study of biotransformations by a crosslinked gamma-lactamase enzyme. Biotechnol. J. 4, 510-516. doi:10.1002/ biot. 200800302

Holt, K. (2004). Biocatalysis and chemocatalysis - a powerful combination for the preparation of enantiomerically pure $\alpha$-amino acids. Pharmachem $3,2-4$. doi:10.1002/chin200542274

Hopmann, K. H., Hallberg, B. M., and Himo, F. (2005). Catalytic mechanism of limonene epoxide hydrolase, a theoretical study. J. Am. Chem. Soc. 127, 14339-14347. doi:10.1021/ja050940p

James, P., Isupov, M. N., Sayer, C., Saneei, V., Berg, S., Lioliou, M., et al. (2014). The structure of a tetrameric $\alpha$-carbonic anhydrase from Thermovibrio ammonificans reveals a core formed around intermolecular disulfides that contribute to its thermostability. Acta Crystallogr. D70, 2607-2618. doi:10.1107/ S1399004714016526

Kobayashi, M., Fujiwara, Y., Goda, M., Komeda, H., and Shimizu, S. (1997). Identification of active sites in amidase: evolutionary relationship between amide bond- and peptide bond-cleaving enzymes. Proc. Natl. Acad. Sci. U.S.A. 94, 11986-11991. doi:10.1073/pnas.94.22.11986

Kong, X.-D., Ma, Q., Zhou, J., Zeng, B.-B., and Xu, J.-H. (2014). A smart library of epoxide hydrolase variants and the top hits for synthesis of $(S)$ $\beta$-blocker precursors. Angew. Chem. Int. Ed. 53, 6641-6644. doi:10.1002/ anie. 201402653

Kotik, M., Archelas, A., and Wohlgemuth, R. (2012). Epoxide hydrolases and their application in organic synthesis. Curr. Org. Chem. 16, 451-482. doi: $10.2174 / 138527212799499840$

Lee, C. K., Daniel, R. M., Shepherd, C., Saul, D., Cary, S. C., Danson, M. J., et al. (2007). Eurythermalism and temperature dependence of enzyme activity. FASEB J. 8, 1934-1941. doi:10.1096/fj.06-7265com

Line, K., Isupov, M. N., and Littlechild, J. A. (2004). The crystal structure of a (-) $\gamma$-lactamase from an Aureobacterium species reveals a tetrahedral intermediate in the active site. J. Mol. Biol. 338, 519-532. doi:10.1016/j.jmb.2004.03.001

Littlechild, J., James, P., Novak, H., and Sayer, C. (2013). "Mechanisms of thermal stability adopted by thermophilic proteins and their use in white biotechnology," in Thermophilic Microbes in Environmental and Industrial Biotechnology, Biotechnology of Thermophiles, 2nd Edn, eds Satyanarayana T., Littlechild J., and Kawarabayasi Y. (London: Springer Publishers), 481-507.

Nestl, B. M., Hammer, S. C., Nebel, B. A., and Hauer, B. (2014). New generation of biocatalysts for organic synthesis. Angew. Chem. Int. Ed. 53, 3070-3095. doi:10.1002/anie.201302195

Ngamsom, B., Hickey, A. M., Greenway, G. M., Littlechild, J. A., Watts, P., and Wiles, C. (2010). Development of a high throughput screening tool for biotransformations utilising a thermophilic L-aminoacylase enzyme. J. Mol. Catal. B Enzym. 63, 81-86. doi:10.1016/j.molcatb.2009.12.013

Novak, H., and Littlechild, J. (2013). "Marine enzymes for biocatalysis. Sources, biocatalytic characteristics and bioprocesses of marine enzymes," in Marine Enzymes with Applications for Biosynthesis of Fine Chemicals, ed. Trincone A. (Cambridge: Woodhead Publishing Series in Biomedicine), 89-102.

Novak, H. R., Sayer, C., Panning, J., and Littlechild, J. A. (2013a). Characterisation of an L-haloacid dehalogenase from the marine psychrophile Psychromonas ingrahamii with potential industrial application. Mar. Biotechnol. 6, 695-705. doi:10.1007/s10126-013-9522-3

Novak, H. R., Sayer, C., Isupov, M. N., Paszkiewicz, K., Gotz, D., Mearns-Spragg, A., et al. (2013b). Marine Rhodobacteraceae L-haloacid dehalogenase contains a novel His/Glu dyad which could activate the catalytic water. FEBS J. 280, 1664-1680. doi:10.1111/febs. 12177
Riley, M., Staley, J. T., Danchin, A., Wang, T. Z., Brettin, T. S., Hauser, L. J., et al. (2008). Genomics of an extreme psychrophile, Psychromonas ingrahamii. BMC Genomics 9:210. doi:10.1186/1471-2164-9-210

Rye, C. A., Isupov, M. N., Lebedev, A. A., and Littlechild, J. A. (2007). An order-disorder twin crystal of L-2-haloacid dehalogenase from Sulfolobus tokodaii. Acta Crystallogr. D63, 926-930. doi:10.1107/S0907444907026315

Rye, C. A., Isupov, M. N., Lebedev, A. A., and Littlechild, J. A. (2009). Biochemical and structural studies of a L-haloacid dehalogenase from the thermophilic archaeon Sulfolobus tokodaii. Extremophiles 13, 179-190. doi:10.1007/ s00792-008-0208-0

Sayer, C., Bommer, M., Ward, J. M., Isupov, M. N., and Littlechild, J. A. (2012). Crystal structure and substrate specificity of the thermophilic serine:pyruvate aminotransferase from Sulfolobus solfataricus. Acta Crystallogr. D68, 763-772. doi:10.1107/S0907444912011274

Sayer, C., Isupov, M. N., Bonch-Osmolovskaya, E., and Littlechild, J. A. (2015). Structural studies of a thermophilic esterase from a new Planctomycetes species, Thermogutta terrifontis. FEBS J. 282, 2846-2857. doi:10.1111/febs.13326

Silverman, D. N., and Lindskog, S. (1988). The catalytic mechanism of carbonic anhydrase: implications of a rate-limiting protolysis of water. Acc. Chem. Res. 21, 30-36. doi:10.1021/ar00145a005

Singleton, M., Isupov, M., and Littlechild, J. (1999). X-ray structure of pyrrolidone carboxyl peptidase from the hyperthermophilic archaeon Thermococcus litoralis. Structure 7, 237-244. doi:10.1016/S0969-2126(99)80034-3

Smith, K. S., Jakubzick, C., Whittam, T. S., and Ferry, J. G. (1999). Carbonic anhydrase is an ancient enzyme widespread in prokaryotes. Proc. Natl. Acad. Sci. U.S.A. 96, 15184-15189. doi:10.1073/pnas.96.26.15184

Tanimoto, K., Higashi, N., Nishioka, M., Ishikawa, K., and Taya, M. (2008). Characterization of thermostable aminoacylase from hyperthermophilic archaeon Pyrococcus horikoshii. FEBS J. 275, 1140-1149. doi:10.1111/j.1742-4658.2008.06274.x

Taylor, S. J. C., McCague, R., Wisdom, R., Lee, C., Dickson, K., Ruecroft, G., et al. (1993). Development of the biocatalytic resolution of 2-azabicyclo [2.2.1] hept-5-en-3-one as an entry to single-enantiomer carbocyclic nucleosides. Tetrahedron 4, 1117-1128. doi:10.1016/S0957-4166(00)80218-9

Toogood, H. S., Brown, R. C., Line, K., Keene, P. A., Taylor, S. J. C., McCague, R., et al. (2004). The use of a thermostable signature amidase in the resolution of the bicyclic synthon (rac)- $\gamma$-lactam. Tetrahedron 60, 711-716. doi:10.1016/j. tet.2003.11.064

Toogood, H. S., Hollingsworth, E. J., Brown, R. C., Taylor, I. N., Taylor, S. J., McCague, R., et al. (2002a). A thermostable L-aminoacylase from Thermococcus litoralis: cloning, overexpression, characterization, and applications in biotransformations. Extremophiles 6, 111-122. doi:10.1007/s007920100230

Toogood, H. S., Taylor, I. N., Brown, R. C., Taylor, S. J. C., McCague, R., and Littlechild, J. A. (2002b). Immobilisation of the thermostable L-aminoacylase from Thermococcus litoralis to generate a reusable industrial biocatalyst. Biocatal. Biotransformation 20, 241-249. doi:10.1080/10242420290029472

Trincone, A. (2011). Marine biocatalysts: enzymatic features and applications. Mar. Drugs 9, 478-499. doi:10.3390/md9040478

Van der Ploeg, J., Van Hall, G., and Janssen, D. B. (1991). Characterization of the haloacid dehalogenase from Xanthobacter autotrophicus GJ10 and sequencing of the dhlB gene. J. Bacteriol. 173, 7925-7933.

Widersten, M., Gurell, A., and Lindberg, D. (2010). Structure-function relationships of epoxide hydrolases and their potential use in biocatalysis. Biochim. Biophys. Acta 1800, 316-326. doi:10.1016/j.bbagen.2009.11.014

Yin, D. L., Bernhardt, P., Morley, K. L., Jiang, Y., Cheeseman, J. D., Purpero, V., et al. (2010). Switching catalysis from hydrolysis to perhydrolysis in P. fluorescens esterase. Biochemistry 49, 1931-1942. doi:10.1021/bi9021268

Conflict of Interest Statement: The authors declare that the research was conducted in the absence of any commercial or financial relationships that could be construed as a potential conflict of interest.

Copyright (C) 2015 Littlechild. This is an open-access article distributed under the terms of the Creative Commons Attribution License (CC BY). The use, distribution or reproduction in other forums is permitted, provided the original author(s) or licensor are credited and that the original publication in this journal is cited, in accordance with accepted academic practice. No use, distribution or reproduction is permitted which does not comply with these terms. 